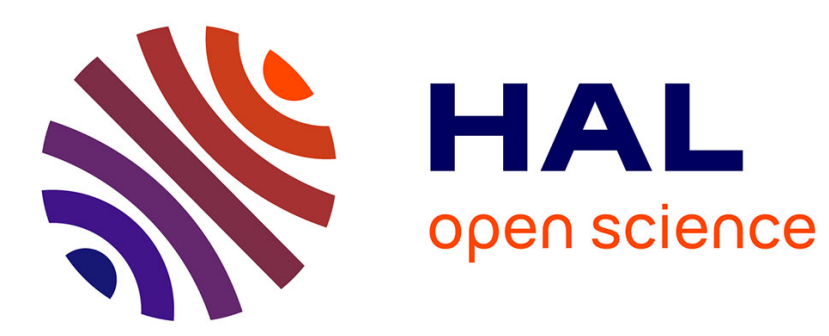

\title{
Representation of preferences over a finite scale by a mean operator
}

Michel Grabisch

\section{To cite this version:}

Michel Grabisch. Representation of preferences over a finite scale by a mean operator. Mathematical Social Sciences, 2006, 52 (2), pp.131-151. 10.1016/j.mathsocsci.2006.05.001 . halshs-00179069

\section{HAL Id: halshs-00179069 \\ https://shs.hal.science/halshs-00179069}

Submitted on 12 Oct 2007

HAL is a multi-disciplinary open access archive for the deposit and dissemination of scientific research documents, whether they are published or not. The documents may come from teaching and research institutions in France or abroad, or from public or private research centers.
L'archive ouverte pluridisciplinaire HAL, est destinée au dépôt et à la diffusion de documents scientifiques de niveau recherche, publiés ou non, émanant des établissements d'enseignement et de recherche français ou étrangers, des laboratoires publics ou privés. 


\title{
Representation of preferences over a finite scale by a mean operator
}

\author{
Michel GRABISCH \\ Université de Paris I - Panthéon-Sorbonne \\ CERMSEM, 106-112 Bd. de l'Hôpital, 75013 Paris, France \\ Tel (+33) 1-44-07-82-85, Fax (+33) 1-44-07-83-01 \\ email Michel.Grabisch@lip6.fr
}

\begin{abstract}
Suppose that a decision maker provides a weak order on a given set of alternatives, each alternative being described by a vector of scores, which are given on a finite ordinal scale $E$. The paper addresses the question of the representation of this weak order by some mean operator, and gives necessary and sufficient conditions for such a representation, with possible shrinking and/or refinement of the scale $E$.
\end{abstract}

Keywords: preference representation, finite scale, mean operator, aggregation of scores, refinement of scale. 


\section{Introduction}

The representation of preferences is a central topic in decision making and measurement theory. Usually, it amounts to finding a real-valued utility function $U$ such that for any pair of alternatives $x, y$ in some set $X$ of alternatives of interest, $x \succsim y$ iff $U(x) \geq U(y)$. When alternatives are $n$-dimensional, i.e., $X=\prod_{i=1}^{n} X_{i}$, a widely studied model is the decomposable model of Krantz et al. [11], where $U$ has the form:

$$
U\left(x_{1} \ldots, x_{n}\right)=G\left(u_{1}\left(x_{1}\right), \ldots, u_{n}\left(x_{n}\right)\right)
$$

where the $u_{i}$ 's are real-valued functions. Assuming that $\succsim$ is a weak order on $X$, it is known that a representation is possible with $G$ being strictly increasing iff $\succsim$ satisfies independence and $X$ is separable [11]. A similar condition for $G$ being non decreasing was found by Bouyssou and Pirlot, as well as many other results when $\succsim$ is not a weak order [1]. It is to be noted that in measurement theory, only $\succsim$ is supposed to be known, and the marginal utility functions $u_{i}$ 's and $G$ are constructed. In this context, non decreasingness of $G$ appears to be a very natural condition.

Let us address a somewhat different although related problem. Suppose that the set of alternatives is finite and that for each $x \in X$ we know the $u_{i}\left(x_{i}\right)$ 's, in addition to the preference $\succsim$. This situation arises in multicriteria evaluation problems, group decision making, etc.: For each alternative $x$, a score is given on some scale $E$, representing the satisfaction or adequacy of $x$ w.r.t. some criterion or some individual. In real situations, it has to be noted that $E$ is most often a finite scale (e.g., \{ "bad","medium","good" $\}$ ). These scores play the role of the quantities $u_{i}\left(x_{i}\right)$. It remains to find a suitable aggregation function $G$ to aggregate the marginal scores into a single overall score, which represents the preference $\succsim$.

Beside non decreasingness, a natural property for $G$ is internality, which means that the overall score should be comprised between the lowest and the highest marginal scores. Internal and non decreasing aggregation functions are usually called mean operators. Hence our main concern will be the representation of preferences by a mean operator.

Few studies have been done in the context of aggregation functions on finite scales, and to the knowledge of the author, almost none deals with preference representation. Conjunctive and disjunctive (hence not internal) aggregation functions on finite scales 
have been studied in detail by Fodor [6], and by Mas, Torrens et al. [17, 18]. Their result are limited since they consider aggregation functions as mappings from $E^{n}$ to $E$, which is obviously very limitative as Examples 1 and 2 will show hereafter. On mean operators on finite scales, there exists a fundamental paper by Ovchinnikov [19], followed by Marichal and Mesiar [12, 13], but their point of view is rather different (although complementary of ours) since they are not concerned with preference representation, but with the meaningfulness of means, in the measurement theoretic sense. Concerning this last topic, Rico et al. [20] have given necessary and sufficient conditions for the representation of preference by a Sugeno integral [21, 15], a particular class of mean operators. Again, their results are limitative since $E$ is considered to be fixed. Therefore, the point of view we adopt here to allow a modification of $E$ in order to have a better ability to represent preferences seems to be original, and opens new horizons.

The paper is organized as follows. We set the framework of the study in Section 2 and state the problem in a clear way. Section 3 gives the main representation result, without considering a refinement of the scale, while Section 4 addresses the case where a refinement is performed. Section 5 indicates possible applications and related works, while Section 6 concludes the paper.

\section{Statement of the problem}

\subsection{Framework and notations}

Let $E$ be a finite chain of $k$ elements $e_{1}<e_{2}<\cdots<e_{k}$, and consider $A \subseteq E^{n}, n>1$. Any $a=\left(a_{1}, \ldots, a_{n}\right) \in A$ is the vector of scores of some alternative or object belonging to $X$ (set of potential alternatives), expressing on $E$ some performance, satisfaction, utility, etc. This situation arises, e.g., in multicriteria decision making, group decision making, decision under uncertainty or risk, and when the evaluation of $a$ is given by an interval $\left[a_{1}, a_{2}\right](n=2)$ (see Section 6 for a comment about this case). We suppose that these scores are given beforehand, and are meaningful according to the concerned domain of decision making. As usual, for $a, b \in A, a \leq b$ stands for $a_{i} \leq b_{i}, i=1, \ldots, n$.

We assume that the decision maker can express his/her preferences on $A$ under the 
form of a weak order (transitive and complete) $\succsim$ on $A \times A$. We denote as usual $a \sim b$ if $a \succsim b$ and $b \succsim a$ hold, and $a \succ b$ if $a \succsim b$ and $\neg(b \succsim a)$.

We denote by $A_{1}, \ldots, A_{k^{\prime}}$ the equivalence classes of $\sim$, i.e., $\forall a, b \in A_{i}, a \sim b$, for $i=1, \ldots, k^{\prime}$, which we call indifference classes. We number them in such a way that $\forall a \in A_{i}, \forall a^{\prime} \in A_{j}, a \succ a^{\prime} \Leftrightarrow i>j$.

We call $(A, \succsim, E)$ the decision profile of the decision maker. Throughout the paper, we will assume non triviality of $\succsim$, i.e., there exist $a, b \in A$ such that $a \succ b$.

We introduce useful notations about indifference classes and alternatives. For any class $A_{i}$, we define:

$$
\begin{aligned}
\left\lfloor A_{i}\right. & :=\min _{a \in A_{i}} \min _{j=1}^{n} a_{j} \\
\left.A_{i}\right\rceil & :=\max _{a \in A_{i}} \max _{j=1}^{n} a_{j} .
\end{aligned}
$$

The interval $\left[\left\lfloor A_{i}, A_{i}\right\rceil\right]$ is denoted $\left\lfloor A_{i}\right\rceil$ for simplicity. Note that this interval may reduce to a singleton. In order to avoid cumbersome conditions for some definitions and proofs, we introduce two (fictitious) additional elements $e_{0}$ and $e_{k+1}$ in $E$, such that $e_{0}<e_{1}$ and $e_{k}<e_{k+1}$, and the fictitious classes $A_{0}$ and $A_{k^{\prime}+1}$ (worst and best possible classes), with $\left\lfloor A_{0}\right\rceil=\left\{e_{0}\right\}$, and $\left\lfloor A_{k^{\prime}+1}\right\rceil=\left\{e_{k+1}\right\}$.

Considering two intervals $[a, b],[c, d]$ (possibly reduced to singletons) of $E$, we say that $[a, b]$ is to the left (resp. to the right) of $[c, d]$ if $b<c$ (resp. $a>d$ ). This is denoted as $[a, b] \lessdot[c, d]($ resp. $[a, b]>[c, d])$. Lastly, for any interval $I=[a, b]$, we denote by $\sharp[a, b]$ or $|I|$ the number of elements in interval $[a, b]$, and denote the bounds by $\lfloor I:=a$, and $I\rfloor:=b$. This notation is extended to the span of alternatives $a \in A$ (i.e. the interval $\left.\left[\min _{i} a_{i}, \max _{i} a_{i}\right]\right)$ by $\left\lfloor a:=\min _{i=1}^{n} a_{i}\right.$ and $\left.a\right\rfloor:=\max _{i=1}^{n} a_{i}$. Following the above convention, the span is denoted by $\lfloor a\rfloor$.

\subsection{The representation problem}

Generally speaking, a representation of a weak order $\succsim$ on the set of potential alternatives $E^{n}$ amounts to finding a mapping $u$ from $E^{n}$ to some chain $C$ endowed with a total order $\leq$, such that $a \succsim b$ if and only if $u(a) \geq u(b)$.

The simplest representation in our case would be to take a function $G: E^{n} \rightarrow E$ satisfying the above, i.e., $C=E$ and $u=G$. The finiteness of $E$ makes the task difficult 
since there may be not enough elements in $E$ to perform the representation. Evidently, this case arises when $k^{\prime}$, the number of indifference classes, is greater than $k$, the number of degrees of the scale. The situation is getting worse if one imposes some natural conditions on $G$, for example internality, which means that the value of $G(a)$ should be comprised between the maximum and minimum scores of $a$. The following example, where $k^{\prime} \leq k$, illustrates this.

EXAMPLE 1: A school director has to evaluate students on several subjects. We consider only 2 subjects for simplicity, say mathematics and literature. Four students $a, b, c, d$ are evaluated on the 2 subjects by the respective professors, using an ordinal scale $\{\alpha, \beta, \gamma, \delta, \epsilon\}$ ( $\alpha$ corresponds to "excellent", $\beta$ to "good", and so forth). Finally, the following evaluation result is given to the director.

\begin{tabular}{|c|c|c|}
\hline student & mathematics & literature \\
\hline$a$ & $\alpha$ & $\alpha$ \\
$b$ & $\alpha$ & $\beta$ \\
$c$ & $\beta$ & $\alpha$ \\
$d$ & $\alpha$ & $\gamma$ \\
\hline
\end{tabular}

After some thought, the director decided that $a$ is the best, then $b$, while $c$ and $d$ are indifferent and worse than $b$ (complete weak order). The problem is however to give them an overall evaluation, reflecting the ranking. Obviously, $a$ should receive $\alpha$ since he/she has $\alpha$ on the two subjects. Then, $b$ should receive at most $\beta$, but no less since his/her scores are at least $\beta$. Then a problem occurs since $c$ should receive at most $\gamma$, but this is strictly lower than his/her lowest score, and this will make him/her complain.

Obviously, the preference of the director cannot be represented on the scale $E$. But nothing prevents the director from creating "half-degrees", like $\alpha^{-}$or $\beta^{+}$. Then $b$ could receive, say $\beta^{+}$, and $c, d$ could receive both $\beta$.

The example shows that an issue to the problem is to make a refinement of the scale $E$. We say that $E^{\prime}$ refines $E$ if $E^{\prime} \supseteq E$ and $E^{\prime}$ is a finite chain. It is important to stress here 
that the refinement of the scale is intended only for the overall score: marginal scores $a_{1}, \ldots, a_{n}$ remains unchanged (they have been given once for all).

Let us put aside the question of refinement, and suppose for the time being that we have as many degrees as we want. What are the conditions on $\succsim$ so that a representation can be done by some particular class of functions $G$, like internal functions, or more generally by what is usually called mean operators? Let us introduce some usual classes of mean operators.

Definition 1 Let $G: E^{n} \longrightarrow E$. We say that $G$ is:

(0) weakly monotone if $\forall a, a^{\prime} \in E^{n},\left\lfloor a \geq a^{\prime}\right\rfloor$ implies that $G(a)<G\left(a^{\prime}\right)$ cannot occur.

(i) internal if $\forall a \in E^{n},\lfloor a \leq G(a) \leq a\rfloor$;

(ii) non decreasing if $\forall a, a^{\prime} \in E^{n}, a \geq a^{\prime}$ implies $G(a) \geq G\left(a^{\prime}\right)$;

(iii) unanimously increasing if it is non decreasing, and $\forall i \in\{1, \ldots, n\}, a_{i}>a_{i}^{\prime}$ implies $G(a)>G\left(a^{\prime}\right)$

(iv) (strictly) increasing if $\forall a, a^{\prime} \in E^{n}, a \geq a^{\prime}$ and $a_{i}>a_{i}^{\prime}$ for at least one $i \in\{1, \ldots, n\}$ imply $G(a)>G\left(a^{\prime}\right)$.

The operator is said to be a mean in the weak sense or a weak mean operator for short if only (i) holds, a mean operator if (i) and (ii) hold, a unanimously increasing mean if (i) and (iii) hold, and a strict mean if (i) and (iv) hold.

Internality means that the overall evaluation should not be beyond the range of the scores, while non decreasingness ensures that an improvement of one score cannot decrease the overall score. One of the first to introduce the term "mean" (in the weak sense) seems to be Cauchy [2]. Note that internality implies weak monotonicity, but not the converse. Likewise, we introduce properties on the decision profile.

Definition 2 Let $A \subseteq E^{n}$ and $\succsim$ be a weak order on $A$. The decision profile $(A, \succsim, E)$ is

(i) weakly coherent if there is no $a, b$ in $A$ such that $a \succ b$ and $a\rfloor \leq\lfloor b$; 
(ii) coherent if for no pair of alternatives $a, b$, we have both $a \succ b$ and $a \leq b$ (or equivalently, $a \geq b$ implies $a \succsim b)$;

(iii) strongly coherent if it is coherent, and for $a, b \in A, a_{i}>b_{i}$ for $i=1, \ldots, n$ implies $a \succ b$;

(iv) strictly coherent if for $a, b \in A, a \geq b$ and $a_{i}>b_{i}$ for at least one $i$ in $\{1, \ldots, n\}$, imply $a \succ b$.

Obviously, strict coherence implies strong coherence, which implies coherence, which in turn implies weak coherence. Remark that if we impose only coherence, we may have $a$ strictly greater than $b$ and $a \sim b$. Conditions (ii), (iii) and (iv) are sometimes called monotonicity, weak Pareto and strong Pareto conditions respectively.

The above two definitions are strongly related, as shown in the following lemma.

Lemma 1 Let $(A, \succsim, E)$ be a weakly coherent decision profile, and $G$ on $E^{n}$ be a weak mean representing $\succsim$. Then the two following propositions are equivalent:

(i) $(A, \succsim, E)$ is coherent (resp. strongly coherent, strictly coherent)

(ii) $G_{\mid A}$ is a mean operator (resp. a strong mean, a strict mean), where $G_{\mid A}$ means the restriction of $G$ to $A$.

Proof: Let us define the weak order $\succsim_{G}$ on $A$ by $a \succsim_{G} b$ iff $G(a) \geq G(b)$. Saying that $G$ represents $\succsim$ means that $\succsim=\succsim_{G}$. Observe that $G_{\mid A}$ being non decreasing (resp. unanimously increasing, strictly increasing) is equivalent to say that $\left(A, \succsim_{G}, E\right)$ is coherent (resp. strongly coherent, strictly coherent). By definition of a mean operator (resp. strong mean, strict mean), the result follows.

The lemma shows that we only have to focus on weak means and weak coherence, other cases will follow immediately. In order to understand the essence of the relation between weak coherence and weak means, let us still suppose that we have as many degrees as we want. Formally, this amounts to say that $E$ is "dense", in the sense that there is no hole in $E$. A hole is a pair $(x, y) \in E^{2}$ with $x<y$ such that there is no $z$ satisfying $x<z<y$. 
Theorem 1 Let us consider $E$ to be dense, and $A \subseteq E^{n}$ to be countable. A profile $(A, \succsim, E)$ is representable by a weak mean operator (resp. a mean operator, a strong mean, a strict mean) if and only if it is weakly coherent (resp. coherent, strongly coherent, strictly coherent), and for any indifference class $A_{j}$, any $a, b \in A_{j}$, a and $b$ have no disjoint spans.

Proof: $\Rightarrow$ ) Assume there exists a weak mean operator $G$ representing $\succsim$, i.e., using previous notations $\succsim_{G}=\succsim$. Internality of $G$ implies weak monotonicity, which in turn is equivalent to weak coherence of $\succsim_{G}$, hence to weak coherence of $\succsim$. Now, for any $a, b \in A_{j}$, we have $G(a)=G(b)$, and due to internality, $a$ and $b$ cannot have disjoint spans.

Using Lemma 1, the other cases follow immediately.

$\Leftarrow)$ Assume the profile is weakly coherent. Since $A$ is countable, for each $a \in A$, we can choose $x_{a} \in\lfloor a\rfloor$ and let $G(a):=x_{a}$. Then $G$ is internal. Since $E$ is dense, and since alternatives in an indifference class have no mutually disjoint span, $x_{a}$ can be chosen so that $a \succsim b$ iff $x_{a} \geq x_{b}$, otherwise there would exist $a, b \in A$ with $a \succ b$ and no $x_{a}$ could be strictly greater than $x_{b}$, which means $\max _{i} a_{i} \leq \min _{i} b_{i}$, which is impossible by weak coherence. Hence $G$ is a weak mean operator representing $\succsim$.

Using Lemma 1, the other cases follow immediately.

This being established, suppose now that the number of degrees in $E$ is limited. Then, as Ex. 1 has shown, the conditions in Th. 1 do not suffice anymore (one can verify that they are fulfilled in Ex. 1: the profile is weakly coherent and there are no alternatives with disjoint spans in indifference classes). Our aim is to find necessary and sufficient conditions for a representation of $\succsim$ on a finite scale.

For the sake of generality, let us come back on the disjoint span condition, and consider the following example, where this condition is not fulfilled.

ExAmple 2: A consumer is asked to espress his/her preference on four cars $a, b, c, d$, knowing their scores on two qualitative criteria, say comfort and safety. The qualitative scale has five degrees: $e_{1}=$ very bad, $e_{2}=\mathrm{bad}$, $e_{3}=$ average, $e_{4}=$ good , and $e_{5}=$ very good . 


\begin{tabular}{|c|c|c|}
\hline car & criterion 1 & criterion 2 \\
\hline$a$ & $e_{1}$ & $e_{1}$ \\
$b$ & $e_{2}$ & $e_{2}$ \\
$c$ & $e_{3}$ & $e_{5}$ \\
$d$ & $e_{5}$ & $e_{4}$ \\
\hline
\end{tabular}

The preference given is $a \sim b \prec c \prec d$ (hence $k^{\prime}=3$ ), so that the profile is coherent. Although there are several solutions for $b, c, d$ (e.g., $G(b)=e_{2}$, $G(c)=e_{3}$ and $\left.G(d)=e_{4}\right)$ which are internal, no internal function $G$ can represent the indifference between $a$ and $b$.

The above example can nevertheless be solved if one "shrinks" elements $e_{1}$ and $e_{2}$ into a single one, say $e_{1}^{\prime}$. Denoting the shrunk scale by $E^{\prime}$ and the shrinking operation by $f: E \longrightarrow E^{\prime}$, a representation on $E^{\prime}$ becomes possible through the function $f \circ G$, taking $G$ as above, and $G(a)=e_{1}, G(b)=e_{2}$. Note that $E^{\prime}$ is nothing else than a partition of $E$. This situation can arise if the scale $E$ contains elements which are in fact indiscernible or non significant for the decision maker.

\subsection{Aim of the paper}

Summarizing the above discussion, the problem we want to address in its full generality can be formulated as follows:

Given a decision profile $(A, \succsim, E)$, with $E$ being a finite chain, under which conditions is it possible to find a representation under the form $u=f \circ G$, with $f: E \longrightarrow E^{\prime}$ a non decreasing mapping defining a partition $E^{\prime}$ of $E$, and $G: E^{n} \longrightarrow E$ is a (weak, strong, strict) mean operator? In case of impossibility, would a refinement of E solve the problem?

Our strategy for tackling this problem will be to find conditions for a representation $f \circ G$ assuming at first no refinement of $E$. Then we will obtain as a simple corollary conditions for a representation where $f$ is not needed (representation by a mean operator), which is the most interesting case in practice. Finally, we will consider a possible refinement of the scale. 
Remark 1: In general there will exist several partitions of $E$ leading to a solution. However, we will consider the finest one (in the sense of the usual order relation on partitions), that is, with a minimum number of shrinks of $E$, as in Ex. 2. Coarser partitions, provided $\left|E^{\prime}\right| \geq k^{\prime}$ may exist, but are to our opinion of little practical interest, since they unnecessarily "blur" the scale.

\section{Representation results without a refinement of the scale}

The following concepts play a central role.

Definition 3 Let $(A, \succsim, E)$ be a decision profile, and $A_{j}$ be some indifference class of $\succsim$, $j \in\left\{1, \ldots, k^{\prime}\right\}$. The core of $A_{j}$ is defined as:

$$
\begin{gathered}
K_{j}:=\emptyset, \text { if } \min _{a \in A_{j}} \max _{i=1}^{n} a_{i}>\max _{a \in A_{j}} \min _{i=1}^{n} a_{i} \\
K_{j}:=\left[\min _{a \in A_{j}} \max _{i=1}^{n} a_{i}, \max _{a \in A_{j}} \min _{i=1}^{n} a_{i}\right], \text { otherwise. }
\end{gathered}
$$

The core is non empty every time there exist two alternatives $a, b$ in $A_{j}$ with disjoint spans (or coinciding on only one point), i.e. such that $\min _{i} a_{i} \geq \max _{i} b_{i}$ (see figure 1 , where the span of three alternatives $a, b, c$ is figured on a 7-elements scale). An important

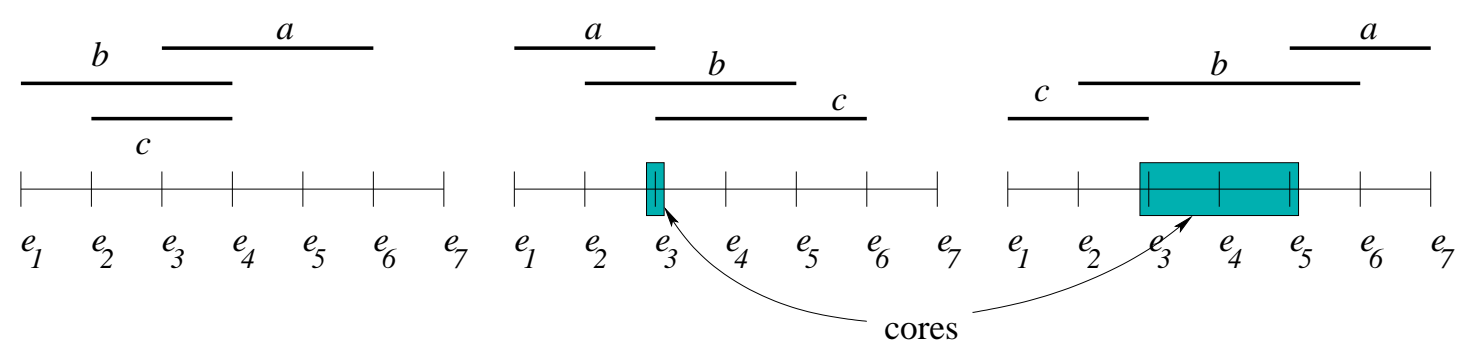

Figure 1: The core of a class: (left) empty core, (center and right) non empty core

remark is that if a class $A_{i}$ is such that $\left\lfloor A_{i}\right\rceil=\left\{e_{l}\right\}$, then its core is the class itself, i.e. $K_{i}=\left\{e_{l}\right\}$. In particular, this is always the case for the fictitious classes $A_{0}$ and $A_{k^{\prime}+1}$. 
Definition 4 Let $(A, \succsim, E)$ be a decision profile. For any indifference class $A_{j}, j=$ $1, \ldots, k^{\prime}$

$$
\begin{aligned}
& \left.\left.A_{>j}\right\rceil:=\min _{j^{\prime}>j} \min _{a^{j^{\prime}} \in A_{j^{\prime}}} a^{j^{\prime}}\right\rfloor \\
& \left\lfloor A_{<j}:=\max _{j^{\prime}<j} \max _{a^{j^{\prime}} \in A_{j^{\prime}}}\left\lfloor a^{j^{\prime}} .\right.\right.
\end{aligned}
$$

Defining the open interval (whenever non empty)

$$
\left.\left\langle A_{j}\right\rangle:=\right]\left\lfloor A_{<j}, A_{>j}\right\rceil[
$$

the interior of $A_{j}$, denoted by $\left\lfloor\stackrel{\circ}{A}_{j}\right\rceil$, is defined by:

$$
\left\lfloor\stackrel{\circ}{A_{j}}\right\rceil:=\left\lfloor A_{j}\right\rceil \cap\left\langle A_{j}\right\rangle .
$$

Figure 2 illustrates the definition, with three classes, and $a, b, c \in A_{1}, d, e$ in $A_{2}$, and $f, g, h \in A_{3}$. The interior of a class indicates the allowable range for scores of any alternative of that class. For example, in Figure $2, e_{9}$ is not in the interior of $A_{2}$, otherwise there would be no available degree in $E$ for the score of $h$, ranked higher than alternatives of class $A_{2}$. Subsequent theorems 2 and 3 will indeed show that the nonemptiness of the interiors is a necessary condition for the existence of a representation. Note that $A_{>k^{\prime}}$ ]

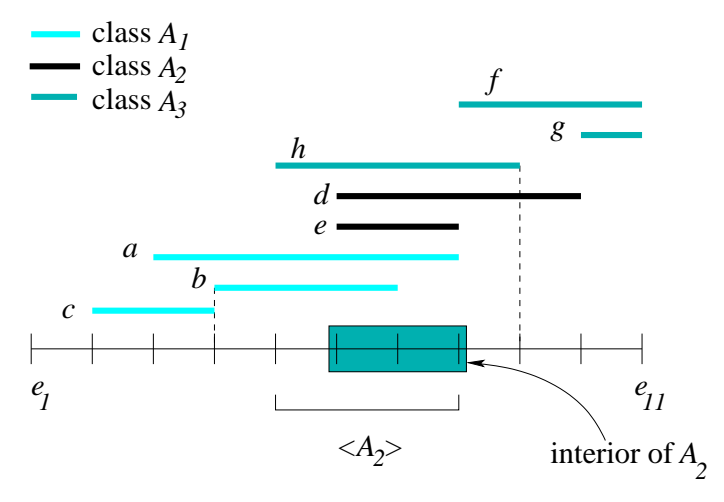

Figure 2: Interior of class $A_{2}$

and $\left\lfloor A_{<1}\right.$ are properly defined thanks to the additional classes $A_{0}$ and $A_{k^{\prime}+1}$. Indeed, $\left\langle A_{1}\right\rangle=\left[e_{1}, A_{>1}\right\rceil\left[\right.$ and $\left.\left\langle A_{k^{\prime}}\right\rangle=\right]\left\lfloor A_{<k^{\prime}}, e_{k}\right]$. Note also that the interior could be empty, even if the decision profile is coherent, as shown by the following simple example. 
ExAmPle 3: Let us consider $n=3$ and 3 alternatives $a, b, c$ such that $c \succ b \succ a$, denoted on a scale with $k=7$, defined in the table below.

\begin{tabular}{|c|c|c|c|}
\hline alternative & criterion 1 & criterion 2 & criterion 3 \\
\hline$a$ & $e_{4}$ & $e_{6}$ & $e_{4}$ \\
$b$ & $e_{4}$ & $e_{7}$ & $e_{4}$ \\
$c$ & $e_{1}$ & $e_{5}$ & $e_{5}$ \\
\hline
\end{tabular}

As it can be verified, the decision profile is coherent, but since $\left\lfloor A_{<2}=e_{4}\right.$ and $\left.A_{>2}\right\rceil=e_{5}$, we have $\left\langle A_{2}\right\rangle=\emptyset$ and thus the interior too is empty (see figure $3)$.

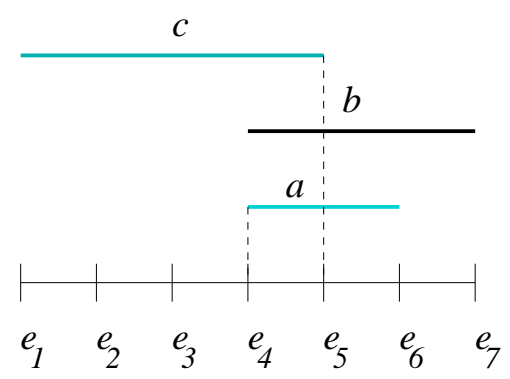

Figure 3: Case of empty interior

We give some properties of the interior, denoting the successor (resp. the predecessor) of an element $e$ on $E$ by $\uparrow e($ resp. $\downarrow e)$.

Lemma 2 Let $(A, \succcurlyeq, E)$ be a decision profile. For any class $A_{j}$, the following properties hold.

(i) $\left.A_{>j}\right\rceil$ and $\left\lfloor A_{<j}\right.$ are non decreasing with $j$, for $j=1, \ldots, k^{\prime}$.

(ii) the intervals $\left\lfloor\stackrel{\circ}{A}_{j}\right\rceil, j=1, \ldots, k^{\prime}$, whenever nonempty are such that $\left\lfloor\stackrel{\circ}{A}_{j}\right.$ and ${\left.\stackrel{\circ}{A_{j}}\right\rceil \text { are }}$ non decreasing with $j$, where $\left\lfloor\stackrel{\circ}{A}_{j}, \stackrel{\circ}{A}_{j}\right\rceil$ are respectively the left and right bounds of $\left\lfloor\stackrel{\circ}{A}_{j}\right\rceil$. Moreover, for any $\left.\left.j^{\prime}<j, A_{j^{\prime}}\right\rceil=A_{j}^{\circ}\right\rceil$ if and only if

$$
\left.\left.\left.\left.A_{>j^{\prime}}\right\rceil=A_{>j}\right\rceil \text { and } A_{j^{\prime}}\right\rfloor \geq \downarrow A_{>j^{\prime}}\right\rceil
$$

while $\left\lfloor\stackrel{\circ}{A}_{j^{\prime}}=\left\lfloor\stackrel{\circ}{A}_{j}\right.\right.$ if and only if

$$
\left\lfloor A_{<j^{\prime}}=\left\lfloorA _ { < j } \text { and } \left\lfloor A_{j} \leq \uparrow\left\lfloor A_{<j}\right. \text {. }\right.\right.\right.
$$


(iii) $\forall j, \forall j^{\prime}<j$ such that $K_{j^{\prime}} \neq \emptyset$ and $\left\lfloor\stackrel{\circ}{A}_{j}\right\rceil \neq \emptyset, K_{j^{\prime}}$ and $\left\lfloor\stackrel{\circ}{A}_{j}\right\rceil$ are disjoint, and the latter is to the right of the former (and symmetrically for $j^{\prime}>j$ ).

(iv) if for some $j^{\prime}<j$ such that $\left\lfloor\stackrel{\circ}{A}_{j}\right\rceil \neq \emptyset,\left\lfloor\stackrel{\circ}{A_{j^{\prime}}}\right\rceil \neq \emptyset$, and $\left\lfloor\stackrel{\circ}{A}_{j}\right\rceil \cap\left\lfloor{\stackrel{\circ}{j^{\prime}}}\right\rceil \neq \emptyset$, then no $a \in A$ can be such that $\lfloor a\rfloor \subseteq\left\lfloor\stackrel{\circ}{A}_{j}\right\rceil \cap\left\lfloor\stackrel{\circ}{A_{j^{\prime}}}\right\rceil$.

(Proof: see appendix)

Let us give some properties of the core and interior when the decision profile is (weakly) coherent.

Lemma 3 If the decision profile $(A, \succcurlyeq, E)$ is weakly coherent, then the non empty cores (if any) are disjoint, and they are ordered the right way, i.e. $K_{j}>K_{j^{\prime}}$ whenever $j>j^{\prime}$.

Proof: Suppose $K_{j^{\prime}} \cap K_{j} \neq \emptyset, j>j^{\prime}$, so that there exists say $e_{l} \in E$ in the intersection. Since $e_{l} \in K_{j^{\prime}}$, there exist $a, b \in A_{j^{\prime}}$ such that $\left.a\right\rfloor \leq e_{l} \leq\left\lfloor b\right.$. Similarly, since $e_{l} \in K_{j}$, there exist $c, d \in A_{j}$ such that $\left.c\right\rfloor \leq e_{l} \leq\lfloor d$. This entails $c\rfloor \leq\lfloor b$. But by definition of $A_{j^{\prime}}, A_{j}$, we have $c \succ b$, which violates weak coherence. Now, $K_{j^{\prime}}$ is to the left of $K_{j}$, otherwise weak coherence will be clearly violated too.

Lemma 4 Let $(A, \succcurlyeq)$ be a weakly coherent decision profile. For any class $A_{j}$, the following properties hold.

(i) $\sharp\left[\left\lfloor A_{<j}, A_{>j}\right]\right]>1$.

(ii) for any $a^{j} \in A_{j}$, if $\left\langle A_{j}\right\rangle \neq \emptyset$, then necessarily $\left\lfloor a^{j}\right\rfloor \cap\left\lfloor\stackrel{\circ}{A_{j}}\right\rceil \neq \emptyset$, otherwise $\sharp\left(\left\lfloor a^{j}\right\rfloor \cap\right.$ $\left.\left[\left\lfloor A_{<j}, A_{>j}\right\rceil\right]\right)=2$.

(iii) if $\left\langle A_{j}\right\rangle \neq \emptyset$, then $\left\lfloor\stackrel{\circ}{A}_{j}\right\rceil \neq \emptyset$.

(iv) if $K_{j} \neq \emptyset$, then $\left\lfloor\stackrel{\circ}{A}_{j}\right\rceil \neq \emptyset$, and $\left\lfloor\stackrel{\circ}{A}_{j}\right\rceil \supseteq K_{j}$.

(Proof: see appendix)

Let us give some results when we want a representation by a (weak) mean operator $G$, i.e., no $f$ is needed. 
Lemma 5 Let $(A, \succsim, E)$ be represented by some weak mean operator $G$. Then:

(i) $G$ is constant over each $A_{i}$.

(ii) Each core $K_{j}$ has at most one element.

Proof: (i) clear.

(ii) Suppose $\left|K_{j}\right|>1$ for some $j$. Then there exist $a, b \in A_{j}$ with disjoint spans. Internality of $G$ implies that necessarily $G(a) \neq G(b)$, hence due to (i), $G$ does not represent $\succsim$, a contradiction.

We are now able to state the main result.

Theorem 2 Let $(A, \succcurlyeq, E)$ be a decision profile with $k^{\prime}$ indifference classes. It exists a representation of $(A, \succcurlyeq, E)$ by $f \circ G$, where $G: E^{n} \longrightarrow E$ is a weak mean operator (resp. a mean operator, a strong mean, a strict mean), and $f$ defines a partition of $E$, if and only if the following conditions are satisfied:

(i) $(A, \succcurlyeq, E)$ is weakly coherent (resp. coherent, strongly coherent, strictly coherent)

(ii) $\forall j, j^{\prime} \in\left\{0, \ldots, k^{\prime}+1\right\}$ such that $j>j^{\prime}$ and $K_{j}, K_{j^{\prime}} \neq \emptyset$,

$$
\sharp\left[K_{j^{\prime}}\right\rfloor,\left\lfloor K_{j}\right] \geq j-j^{\prime}+1
$$

(iii) $\left\lfloor\stackrel{\circ}{A_{j}}\right\rceil \neq \emptyset$ for $j=1, \ldots, k^{\prime}$.

(iv) $\forall j, j^{\prime} \in\left\{1, \ldots, k^{\prime}\right\}$ such that $j>j^{\prime}$,

$$
\left.\sharp\left[\left\lfloor{\stackrel{\circ}{j^{\prime}}}, \stackrel{\circ}{j}\right\rceil\right\rceil\right] \geq j-j^{\prime}+1 .
$$

(Proof: see appendix)

Remark 2: In condition (iii), $\left\lfloor\stackrel{\circ}{A}_{j}\right\rceil$ can be replaced by $\left\langle A_{j}\right\rangle$ (see proof). Also, condition (iii) is a special case of (iv) if we allow $j=j^{\prime}$.

Remark 3: Taking (iv) with $j=k^{\prime}, j^{\prime}=1$, we get $\sharp\left[\left\lfloor\stackrel{\circ}{A_{1},}, \stackrel{\circ}{A_{k^{\prime}}}\right]\right] \geq k^{\prime}$, which entails $k \geq k^{\prime}$. 
Remark 4: Condition (iv) is sound since due to Lemma 2 (ii), interiors are ordered, so that $\left[\left\lfloor\stackrel{\circ}{A}_{j^{\prime}}, \stackrel{\circ}{\left.A_{j}\right\rceil}\right]\right.$ is never empty.

Remark 5: The proof is constructive, and gives all solutions for $G$ (if any), considering that $f$ defines the finest partition (see Remark 1). This partition is very easy to obtain, it is merely the partition obtained by shrinking into a single element all elements in a core, for those having more than one element.

Figure 4 illustrates the theorem. We consider 8 alternatives $a, b, c, d, e, f, g, h, 3$ criteria and a scale $E$ with 9 elements. The decision profile is defined as follows.

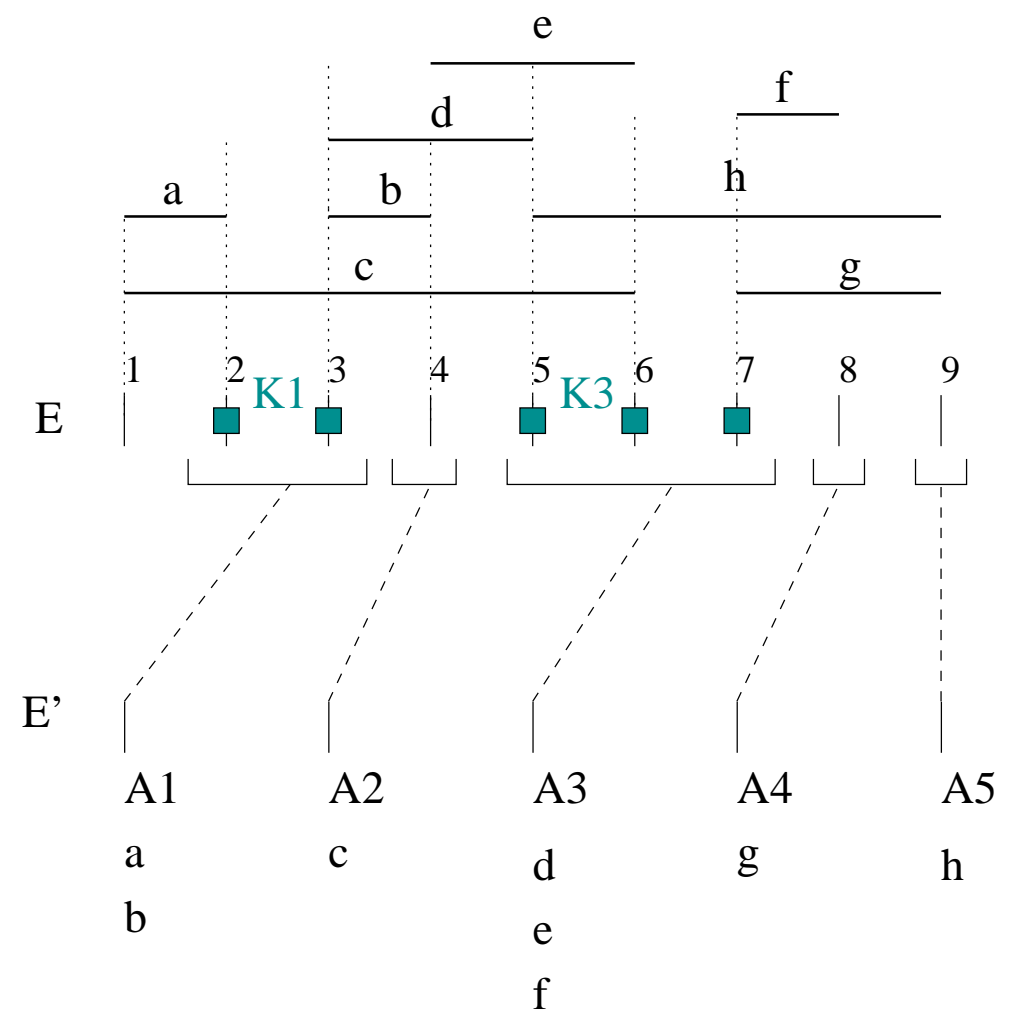

Figure 4: An example of preference representation 


\begin{tabular}{|c|c|c|c|c|}
\hline alternative & criterion 1 & criterion 2 & criterion 3 & class \\
\hline$a$ & $e_{1}$ & $e_{2}$ & $e_{2}$ & $A_{1}$ \\
$b$ & $e_{4}$ & $e_{3}$ & $e_{3}$ & $A_{1}$ \\
$c$ & $e_{1}$ & $e_{1}$ & $e_{6}$ & $A_{2}$ \\
$d$ & $e_{3}$ & $e_{5}$ & $e_{4}$ & $A_{3}$ \\
$e$ & $e_{4}$ & $e_{4}$ & $e_{6}$ & $A_{3}$ \\
$f$ & $e_{8}$ & $e_{7}$ & $e_{7}$ & $A_{3}$ \\
$g$ & $e_{9}$ & $e_{8}$ & $e_{7}$ & $A_{4}$ \\
$h$ & $e_{5}$ & $e_{9}$ & $e_{7}$ & $A_{5}$ \\
\hline
\end{tabular}

Classes are such that $A_{1} \prec A_{2} \prec \cdots \prec A_{5}$. There are two cores $K_{1}$ and $K_{3}$. It can be verified that the profile is coherent, and conditions (ii), (iii) and (iv) of the theorem are satisfied. Hence there exists a mean operator $G$ and a partition of $E$ representing the preference. The finest partition is indicated by the brackets. Note that $e_{1}$ is not used in the representation. Solutions for $G$ are: $G(a)=e_{2}, G(b)=e_{3}, G(c)=e_{4}, G(d)=e_{5}$, $G(e)=e_{5}$ or $e_{6}, G(f)=e_{7}, G(g)=e_{8}$ and $G(h)=e_{9}$. Note that even if $a \sim b, G(a)=e_{2}$ and $G(b)=e_{3}$ is possible since elements $e_{2}$ and $e_{3}$ are shrunk into a single one.

We turn now to the case where no partitioning of $E$ is needed.

Theorem 3 Let $(A, \succcurlyeq, E)$ be a decision profile with $k^{\prime}$ indifference classes. It exists a representation of $(A, \succcurlyeq, E)$ by a weak mean operator (resp. a mean operator, a strong mean, a strict mean) $G$, if and only if the following conditions are satisfied:

(i) $(A, \succcurlyeq, E)$ is weakly coherent (resp. coherent, strongly coherent, strictly coherent)

(ii) $\forall j, j^{\prime} \in\left\{0, \ldots, k^{\prime}+1\right\}$ such that $j>j^{\prime}$ and $K_{j}, K_{j^{\prime}} \neq \emptyset$,

$$
\sharp\left[K_{j^{\prime}}\right\rfloor,\left\lfloor K_{j}\right] \geq j-j^{\prime}+1 .
$$

(iii) $\left\lfloor\stackrel{\circ}{A_{j}}\right\rceil \neq \emptyset$ for $j=1, \ldots, k^{\prime}$.

(iv) $\forall j, j^{\prime} \in\left\{1, \ldots, k^{\prime}\right\}$ such that $j>j^{\prime}$,

$$
\sharp\left[\left\lfloor\stackrel{\circ}{A}_{j^{\prime}}, \stackrel{\circ}{A}_{j}\right\rceil\right] \geq j-j^{\prime}+1 .
$$


(v) $\left|K_{j}\right| \leq 1, j=1, \ldots, k^{\prime}$.

Remark that the additional condition (v) means that in an indifference class, the pairwise intersection of alternatives is never empty. This was the condition we expected.

Proof: $\Rightarrow$ ) Taking $f=I d$, Th. 2 shows that necessarily (i) to (iv) hold. Using Lemma 5 (ii), we deduce condition (v).

$\Leftarrow)$ Using Th. 2, by conditions (i) to (iv), we know that there exists a representation under the form $f \circ G$. Suppose among all possible solutions, there exists one with $G$ being constant over each $A_{j}$. Then a partitioning of $E$ would be useless, and the representation could be achieved by $G$ alone. It suffices then to show that condition (v) implies that it is possible to choose $G$ constant on each $A_{j}$. Refering to the "only if" part of the proof of Th. 2 , we see that the set of possible values for $G(a)$, for $a \in A_{j}$ is the interval $\left[\psi_{j}, \phi_{j}\right]$. We are done if we show that there exists $x_{j} \in\left[\psi_{j}, \phi_{j}\right]$ belonging to the span of each $a \in A_{j}$, hence to $\cap_{a \in A_{j}}\lfloor a\rfloor$, since in this case it suffices to put $G(a)=x_{j}$ for all $a \in A_{j}$.

By the proof of Th. 2, we know that $\left[\psi_{j}, \phi_{j}\right] \cap\lfloor a\rfloor \neq \emptyset$, for all $a \in A_{j}$. Suppose there is no common point of intersection in the interval $\left[\psi_{j}, \phi_{j}\right]$. This would mean that it exist two alternatives $a, a^{\prime} \in A_{j}$ with disjoint spans. But this contradicts the fact that $\left|K_{j}\right| \leq 1$

\section{Refinement of the scale}

Theorem 2 tells us that the representation of preference is not possible with $f \circ G$ when:

- either the decision profile is not coherent in some sense (condition (i) of Th. 2)

- or the scale $E$ has not enough elements (conditions (ii), (iii) and (iv)).

In the first case, we may say that the decision maker exhibits a preference which is incompatible with a mean operator, and so our model cannot work anyway. In the second case, it is just a matter of poorness of the scale, and we can remedy to this.

In this section, we address the second case. We suppose that the decision profile is such that one or several among the conditions (ii) through (iv) does not hold. The aim 
is to build a refined scale $\bar{E}$ from $E$ so that all conditions hold (i.e. the preference is representable). A refinement of $E$ is any scale $\bar{E}$ such that $\bar{E} \supseteq E$.

As we assume that scores of alternatives are given beforehand on $E$ by the decision maker, we cannot change this assignment, and the only element of freedom we have is to assign global scores on $\bar{E}$. Thus, $G$ maps $E^{n}$ to $\bar{E}$, and $f$ performs a partitioning of $\bar{E}$.

It is clear that to fulfill conditions (ii) through (iv), it suffices to add a finite number of elements to $E$. The exact places where to add these elements are dictated by the conditions themselves. The problem to find a minimal number of elements to add requires however a careful study, which is outside the scope of this paper. An algorithm of refinement has been proposed by the author, which gives a minimal refinement, see [9] for details.

In conclusion, one can give the following result, coming directly from Th. 2 and 3 and the above considerations.

Corollary 1 Let $(A, \succsim, E)$ be a decision profile, E being a finite chain.

(i) There exists a representation of $\succsim$ by $f \circ G$, where $G$ is weak mean operator (resp. a mean operator, a strong mean, a strict mean) valued on $\bar{E}, f$ defines a partition of $\bar{E}$, and $\bar{E}$ is a finite refinement of $E$, if and only if the profile is weakly coherent (resp. coherent, strongly coherent, strictly coherent).

(ii) There exists a representation of $\succsim$ by $G$, where $G$ is weak mean operator (resp. a mean operator, a strong mean, a strict mean) valued on $\bar{E}$, where $\bar{E}$ is a finite refinement of E, if and only if the profile is weakly coherent (resp. coherent, strongly coherent, strictly coherent), and for any indifference class $A_{j}$, any $a, b \in A_{j}$, a and $b$ have no disjoint spans.

This result can be seen as a complement of Th. 1, since it gives the minimal requirement for having a representation by a mean operator on a finite scale.

We give a last example to illustrate the refinement process. These results have been obtained from an implementation in $\mathrm{C}$ language of the refinement algorithm in [9] and of the constructive proof of Th. 2 .

ExAmple 4: We consider a list of 11 students labelled from A to K, who are evaluated on 3 subjects (say mathematics, physics and literature), on a 
qualitative scale $E$ with 5 degrees. The degrees of the scale are simply referred to by their number, with 1 being the worst degree and 5 the best one. Table 1 gives the scores obtained by the 11 students. The following ranking has been

\begin{tabular}{|c||c|c|c|}
\hline Student & Math. & Physics & Liter. \\
\hline A & 1 & 1 & 3 \\
B & 1 & 2 & 1 \\
\hline C & 1 & 3 & 2 \\
\hline D & 2 & 2 & 2 \\
E & 3 & 2 & 2 \\
F & 2 & 2 & 5 \\
\hline G & 4 & 4 & 4 \\
H & 4 & 4 & 3 \\
\hline I & 5 & 3 & 3 \\
\hline J & 4 & 3 & 5 \\
\hline K & 5 & 3 & 5 \\
\hline
\end{tabular}

Table 1: Scores obtained by the 11 students

determined by the teachers:

$$
A \sim B \prec C \prec D \sim E \sim F \prec G \sim H \prec I \prec J \prec K .
$$

There are 7 indifference classes, labelled from 1 (worst) to 7 (best). One can verify that the decision profile is coherent. Table 2 gives the span, core and interior of all classes. We remark that the cores are empty or reduced to singletons, hence no partitioning of the scale is needed (Th. 3). However, since empty interiors exist, a refinement of the scale is necessary. It can be checked that a minimal refinement so that all conditions in Th. 3 are satisfied is:

$$
1<1^{\prime}<2<3<4<4^{\prime}<4^{\prime \prime}<5 .
$$




\begin{tabular}{|c||c|c|c|}
\hline Class & Span & Core & Interior \\
\hline 1 & {$[1,3]$} & empty & {$[1,1]$} \\
2 & {$[1,3]$} & empty & empty \\
3 & {$[2,5]$} & {$[2,2]$} & {$[2,3]$} \\
4 & {$[3,4]$} & {$[4,4]$} & {$[3,4]$} \\
5 & {$[3,5]$} & empty & empty \\
6 & {$[3,5]$} & empty & empty \\
7 & {$[3,5]$} & empty & {$[5,5]$} \\
\hline
\end{tabular}

Table 2: Characteristics of the indifference classes

Then it can be checked that the only solution for $G$ is:

$$
\begin{gathered}
G(\mathrm{~A})=G(\mathrm{~B})=1, G(\mathrm{C})=1^{\prime}, G(\mathrm{D})=G(\mathrm{E})=G(\mathrm{~F})=2, G(\mathrm{G})=G(\mathrm{H})=4, \\
G(\mathrm{I})=4^{\prime}, G(\mathrm{~J})=4^{\prime \prime}, G(K)=5 .
\end{gathered}
$$

\section{Applications and related works}

We indicate here in which cases the results we develop could be useful, and mention related research.

In most of applied domains, computing an overall score from individual scores (coming from criteria, experts, etc.) is done through a mean operator (most often the weighted arithmetic mean). The additional restriction we find in social sciences is that often scales are qualitative, and hence finite. This explains why we focus on the representation of preferences by a mean operator on finite scales.

The question arises why a representation $G$ (or $f \circ G$ ) should be useful, considering that this representation could be complex, and that finally the weak order $\succsim$ contains all the information. The representation is useful in at least the following situations. To detail them, we consider that underlying dimensions are criteria.

- Knowing $G$ or $f \circ G$ enables an analysis of the model in terms of importance of criteria, or any other useful index putting into light the behaviour of the decision maker (DM): interaction indices which detect subsets of substitutive and complementary 
criteria [7, 10], veto or favor indices showing which criteria must be necessarily satisfied or which criteria it is sufficient to satisfy [8, 14], "andness/orness" which show if aggregation of scores is done conjunctively (by an "and" operator) or disjunctively (by an "or" operator) [14], etc. Of course, this analysis is possible only if an analytical expression of $G$ is known. Since our results provide only values of $G$ on $A$, to get an analytical form is a fitting problem with some family of mean operators, a topic outside the scope of this paper (see below for more details).

Also, the simple fact to have a representation with $G$ alone or necessarily with $f \circ G$ already gives information on the perception of scales by the DM: if $f$ is necessary, then some degrees are useless or indiscernable by the DM. In summary, the aim is to analyse the observed preference or to give an explanation about the preference shown by the DM. In interactive decision systems, it is mandatory to be able to explain the preference or the decision made.

- Most of the time, the size of the set of alternatives $A$ is kept small in order to avoid any burden on the DM, and $A$ should contain what is called prototypical alternatives. Hence the preference is far to be known on the whole set of potential alternatives $E^{n}$. But having a representation in an analytical form, it is now possible to compute an overall score for all potential alternatives, and thus to extend $\succsim$ on $E^{n}$. Hence, it is possible to predict the behaviour of the DM on any new alternative.

- Even if no analytical form of $G$ is available, knowing the values $G(a)$ for alternatives $a \in A$ is more informative than knowing $\succsim$ on $A$, if some semantics is attached to the elements of E, like in Ex. 1 and 2. Particularly in Ex. 1, it could be of importance to know if a given student has achieved an average score at least as good as, e.g., $\gamma=$ fair, since "fair" could be the minimum required score for receiving the diploma. Analysis/explanation and prediction are very common in multicriteria decision making, but in a numerical context. Our results provide the first necessary steps to do the same in an ordinal context. We recall that the proof of theorems 2 and 3 are constructive and give all solutions (when they exist) for $f \circ G$ restricted on $A$, and that we have an algorithm of refinement of scales providing minimal refinements when the scale is too coarse (see Ex. 4) [9]. The extension of $G$ (or $f \circ G$ ) from $A$ to $E^{n}$ is just a matter of 
choosing a family of operators, and to search for those operators which fit the best on $A$. A family of operators which is of particular interest is provided by the so-called Sugeno integral [21], which is a mean operator, not strong in general. As shown by Marichal [16], they coincide with lattice polynomials $P\left(x_{1}, \ldots, x_{n}\right)$ which are non decreasing w.r.t all arguments, and such that $P(0, \ldots, 0)=0, P(1, \ldots, 1)=1$, hence they cover a wide range of ordinal operators.

We address now related works. As said in the introduction, there are few studies related to aggregation functions on finite scales (see Rico et al. [20]), but we think that our approach can be linked to interval orders, and to qualitative models of preference.

Rico et al. [20] have found necessary and sufficient conditions for the existence of a Sugeno integral representing a given complete weak order on a set of alternatives $A$. Our study both completes and can benefit from these results, since on one hand we provide general results about any kind of mean operators, and we allow for scale refinement or shrinking, a feature which is not in the study of Rico et al., and on the other hand, their results can be readily applied once we have done the necessary refinements and shrinkings of the scale, to see if a Sugeno integral is a possible candidate for a representation.

Concerning interval orders, we notice that weak coherence, as well as the notion of weak mean operator, involve only the boundaries of the span of alternatives, not the scores individually. In other words, these notions can be used as well when the evaluation of an alternative $a$ is mono-dimensional, but interval-valued. Let us denote by $[\underline{a}, \bar{a}]$ the interval in $E$ assigned to alternative $a$. We know that (see e.g. Fishburn [5]) $\succsim$ is a complete interval order iff it can be represented by intervals, such that

$$
\begin{aligned}
& \text { - } a \succ b \Leftrightarrow[\underline{a}, \bar{a}]>[\underline{b}, \bar{b}] \\
& \text { - } a \sim b \Leftrightarrow[\underline{a}, \bar{a}] \cap[\underline{b}, \bar{b}] \neq \emptyset .
\end{aligned}
$$

Any interval order can be represented by a function and a variable threshold.

The difference with our model is that we assume to have a complete weak order on intervals, but strict preference is less restrictive than the above condition, since we just require weak coherence, i.e. if $a \succ b$ then we can have neither $[\underline{a}, \bar{a}] \lessdot[\underline{b}, \bar{b}]$ nor $\bar{a}=\underline{b}$. Then Theorem 2 tells that such a weak order can be represented by a weak mean operator 
(assuming there is enough elements in $E$ ), in other words, each interval is represented by a single number contained in the interval.

Concerning qualitative models of preference representations, a great deal of work has been achieved in the framework of decision under uncertainty, trying to adapt the Savagian model to a qualitative finite framework (see, e.g., [3], and more recently [4]). The representation is done through a Sugeno integral in [3], while Fargier and Sabbadin in [4] advocates the use of a lexicographic version of Sugeno integral, in order to have a better representation power (for example, the Sugeno integral can never be a strict mean). Fargier and Sabbadin show that their lexicographic model can equivalently be represented by a weighted arithmetic mean after a suitable mapping of the qualitative finite scale into the real line. This is undoubtly close to our view, although many assumptions and aims in our respective models differ.

\section{Concluding remarks}

The philosophy of the paper and main results can be summarized as follows:

- Suppose that for representing a decision profile, you have at disposal as many degrees in $E$ as you want. What are the relations between the various type of means, and various types of coherence of the decision profile? This is given in Th. 1.

- Suppose that you have a limited scale $E$. Is the decision profile representable on $E$ by a suitable mean operator (whose type is known from Th. 1)? If interiors of indifference classes are nonempty and are enough large in case of overlapping, and if cores (if any) are enough far from each others, then a representation is possible, with perhaps a shrinking of the scale $E$. This Th. 2. If in addition there is no pairwise disjoint spans of alternatives in indifference classes, then no shrinking is necessary. This is Th. 3 .

- Suppose that the representation is not possible because there is not enough degrees. Then add degrees in $E$ so that conditions (ii), (iii) and (iv) of Th. 2 are fulfilled. This is Section 4. 
To finish, we would like to indicate future study. An interesting topic for future research would be to find similar conditions for representation in the case of other aggregation operators, or more specific ones. In the context of ordinal aggregation, we know from Ovchinnikov [19] that the only meaningful (in the sense of measurement theory) mean operators on ordinal scales are the operators returning one of the input scores. This is clearly a particular class of our mean operators, and it would be interesting to know under which conditions on the decision profile a representation by such a meaningful mean is possible.

\section{Acknowledgement}

This paper has benefited from many discussions with D. Bouyssou, Ch. Labreuche, T. Marchant, M. Pirlot, P. Wakker, who are deeply acknowledged. We thank also the anonymous reviewers for their useful suggestions. 


\section{Proofs of lemmas and Theorem 2}

\section{Proof of Lemma 2}

(i) for any $j_{1}<j_{2}$ :

$$
\begin{aligned}
& \max _{j^{\prime}<j_{1}} \max _{a^{j^{\prime}} \in A_{j^{\prime}}}\left\lfloor a^{j^{\prime}} \leq \max _{j^{\prime}<j_{2}} \max _{a^{j^{\prime}} \in A_{j^{\prime}}}\left\lfloor a^{j^{\prime}}\right.\right. \\
& \left.\left.\min _{j^{\prime}>j_{1}} \min _{a^{j^{\prime}} \in A_{j^{\prime}}} a^{j^{\prime}}\right\rfloor \leq \min _{j^{\prime}>j_{2}} \min _{a^{j^{\prime}} \in A_{j^{\prime}}} a^{j^{\prime}}\right\rfloor .
\end{aligned}
$$

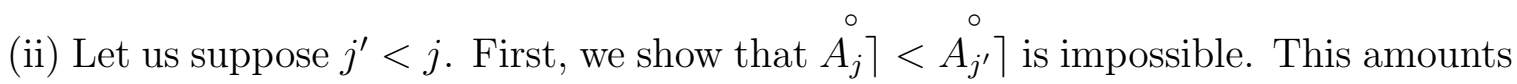
to show that

$$
\left.\left.\left.\left.\min \left(A_{j}\right\rceil, \downarrow A_{>j}\right\rceil\right)<\min \left(A_{j^{\prime}}\right\rceil, \downarrow A_{>j^{\prime}}\right\rceil\right)
$$

is impossible. We observe that $\left.\left.\downarrow A_{>j^{\prime}}\right\rceil<A_{j}\right\rceil$ (simply remark that $\left.\left.A_{>j^{\prime}}\right\rceil \leq A_{j}\right\rceil$ ). Also by Lemma 2 (i), $\left.\left.A_{>j^{\prime}}\right\rceil \leq A_{>j}\right\rceil$.

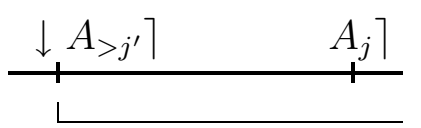

possible positions of $\left.\downarrow A_{>j}\right\rceil$

Then if $\left.\left.\left.\downarrow A_{>j}\right\rceil \in\left[\downarrow A_{>j^{\prime}}\right\rceil, A_{j}\right\rceil\right]$, the above inequality becomes $\left.\downarrow A_{>j}\right\rceil<\min \left(A_{j^{\prime}}\right\rfloor, \downarrow$ $\left.A_{>j^{\prime}}\right\rceil$ ), which is impossible (see the above figure). If on the contrary $\left.\left.\downarrow A_{>j}\right\rceil>A_{j}\right\rceil$, the above inequality becomes $\left.\left.\left.A_{j}\right\rceil<\min \left(A_{j^{\prime}}\right\rceil, \downarrow A_{>j^{\prime}}\right\rceil\right)$, which is also impossible. Necessary and sufficient conditions to have equality come from the examination of the figure.

Using the same way, we show that $\stackrel{\circ}{A}_{j}<\left\lfloor\stackrel{\circ}{A}_{j^{\prime}}\right.$ is impossible. This amounts to show that

$$
\max \left(\left\lfloorA_{j}, \uparrow\left\lfloor A_{<j}\right)<\max \left(\left\lfloorA_{j^{\prime}}, \uparrow\left\lfloor A_{<j^{\prime}}\right)\right.\right.\right.\right.
$$

is impossible. We observe that $\uparrow\left\lfloor A_{<j}>\left\lfloor A_{j^{\prime}}\right.\right.$. Also, by Lemma 2 (iv), $\left\lfloor A_{<j^{\prime}} \leq\left\lfloor A_{<j}\right.\right.$. Then if $\uparrow\left\lfloor A_{<j^{\prime}} \in\left[\left\lfloor A_{j^{\prime}}, \uparrow\left\lfloor A_{<j}\right]\right.\right.\right.$, the above inequality becomes impossible, the same for the other case.

(iii) We suppose $K_{j^{\prime}} \neq \emptyset, j^{\prime}<j$. Let us call $a \in A_{j^{\prime}}$ an element such that $\left\lfloor a=K_{j^{\prime}}\right\rfloor$ (which exists since the core is non empty). From the definition, we have:

$$
\left\lfloor A_{<j} \geq\left\lfloor a=K_{j^{\prime}}\right\rfloor\right.
$$


which implies $\uparrow\left\lfloor A_{<j}>\left\lfloor a\right.\right.$, so that $\left\langle A_{j}\right\rangle$ (and consequently $\left\lfloor\stackrel{\circ}{A}_{j}\right\rceil$ ) is to the right of $K_{j^{\prime}}$.

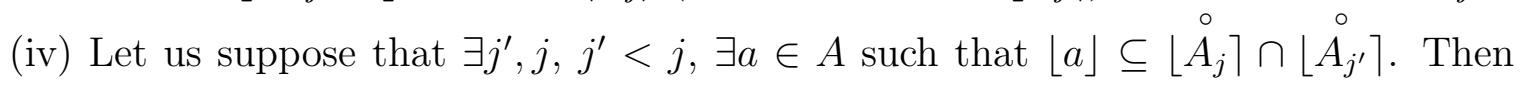
either $a$ belongs to $A_{j^{\prime \prime}}, j^{\prime \prime}<j$, or $a$ belongs to a class $A_{j^{\prime \prime}}$, with $j^{\prime \prime}>j^{\prime}$. In the first case, $\left\lfloor A_{<j} \geq\left\lfloor a\right.\right.$, which means that $\lfloor a\rfloor$ cannot be included in $\left\lfloor A_{j}\right\rceil$, hence not in the intersection too. In the second case, $\left.\left.A_{>j^{\prime}}\right\rfloor \leq a\right\rfloor$, which means that $\lfloor a\rfloor$ cannot be included into $\left\lfloor A_{j^{\prime}}\right\rceil$.

\section{Proof of Lemma 4}

For any $A_{j}$ let us denote by $a^{>j}$ and $a^{<j}$ the alternatives (possibly non unique) corresponding to the bounds of $\left\langle A_{j}\right\rangle$, i.e. such that $\left.\left.a^{>j}\right\rfloor=A_{>j}\right\rceil$, and $\left\lfloor a^{<j}=\left\lfloor A_{<j}\right.\right.$.

(i) Suppose that $\left\lfloor A_{<j} \geq A_{>j}\right\rceil$. This implies that $\left\lfloor a^{<j} \geq a^{>j}\right\rfloor$. But $a^{<j} \prec a^{>j}$, and the weak coherence assumption is violated. Thus, $\left\lfloor A_{<j}<A_{>j}\right\rceil$.

(ii) Since $A_{j}$ is nonempty, there exists $a \in A_{j}$, and in particular $a \prec a^{>j}$ and $a \succ a^{<j}$. Due to the weak coherence assumption, we cannot have $\left\lfloor a \geq a^{>j}\right\rfloor$. Similarly, $\left.a\right\rfloor \leq\left\lfloor a^{<j}\right.$ is also impossible. This entails $\lfloor a\rfloor \cap\left\langle A_{j}\right\rangle \neq \emptyset$ when $\left\langle A_{j}\right\rangle \neq \emptyset$, and since $\lfloor a\rfloor \subseteq\left\lfloor A_{j}\right\rfloor$, the

result holds. Now if $\left\langle A_{j}\right\rangle=\emptyset$, then we know from lemma 4 (i) that $\sharp\left[\left\lfloor A_{<j}, A_{>j}\right\rceil\right]=2$. Then it is easy to check that the result holds.

(iii) We have to prove that $\left\lfloor A_{j}\right\rceil \cap\left\langle A_{j}\right\rangle \neq \emptyset$. Due to lemma 4 (ii), we know that $\forall a \in A_{j},\lfloor a\rfloor \cap\left\langle A_{j}\right\rangle \neq \emptyset$. Since $\left\lfloor a_{j}\right\rfloor \subseteq\left\lfloor A_{j}\right\rceil$, the result holds.

(iv) Assume that $\left.\left.K_{j}\right\rfloor \geq A_{>j}\right\rceil$. Let $a \in A_{j}$ such that $\left\lfloor a \geq K_{j}\right\rfloor$. We have $\left\lfloor a \geq a^{>j}\right\rfloor$, but $a \prec a^{>j}$, which violates weak coherence. Doing the same on the left side, we conclude that $\left.\left.K_{j}\right\rfloor<A_{>j}\right\rceil$ and $\left\lfloor K_{j}>\left\lfloor A_{<j}\right.\right.$. Since $K_{j} \neq \emptyset$, this entails $\sharp\left[\left\lfloor A_{<j}, A_{>j}\right\rceil\right] \geq 3$, i.e. $\left\langle A_{j}\right\rangle \neq \emptyset$, and by Lemma 4 (iii), $\left\lfloor\stackrel{\circ}{A}_{j}\right\rceil \neq \emptyset$. Also $K_{j} \subseteq\left\langle A_{j}\right\rangle$, and since $K_{j} \subseteq\left\lfloor A_{j}\right\rceil$, we get $K_{j} \subseteq\left\lfloor\stackrel{\circ}{A_{j}}\right\rceil$.

\section{Proof of Theorem 2}

$\Rightarrow$ ) Let $f \circ G$ represents $\succsim$, with $G$ being a weak mean and show that (i) to (iv) hold. To show this, it suffices to assume $f=I d$.

(i) If $(A, \succsim, E)$ is not weakly coherent, then there exist $a^{\prime} \in A_{j^{\prime}}$ and $a \in A_{j}, j^{\prime}<j$, and $\left\lfloor a^{\prime} \geq a\right\rfloor$. Since $G$ is a weak mean, this entails $G\left(a^{\prime}\right) \geq G(a)$, so that the representation 
condition does not hold.

(ii) Suppose $j^{\prime}<j$ such that $K_{j^{\prime}}, K_{j} \neq \emptyset$. There are $j-j^{\prime}+1$ classes between $A_{j^{\prime}}$ and $A_{j}$ (including them). By definition of the core, there exist $a^{j}, a^{j^{\prime}}$ in $A_{j}, A_{j^{\prime}}$ respectively such that $\left.K_{j^{\prime}}\right\rfloor=\left\lfloor a^{j^{\prime}}\right.$ and $\left\lfloor K_{j}=a^{j}\right\rfloor$. At best $G$ will be such that $G\left(a^{j^{\prime}}\right)=\left\lfloor a^{j^{\prime}}\right.$ and $G\left(a^{j}\right)=a^{j}$, so that we need at least $j-j^{\prime}-1$ elements between cores $K_{j}, K_{j^{\prime}}$ to rank classes $A_{j^{\prime}+1}, \ldots, A_{j-1}$.

(iii) From Lemma 4 (i), we know that $\sharp\left[\left\lfloor A_{<j}, A_{>j}\right\rceil\right]>1$. Let us show that the case $\sharp\left[\left\lfloor A_{<j}, A_{>j}\right\rceil\right]=2$ is impossible. Suppose it holds (this is indeed the situation of Ex. 1 . Then it means that $\left\lfloor a^{<j}=\downarrow a^{>j}\right\rfloor$. At best $G$ will assign to $a^{<j}$ and $a^{>j}$ the elements $\left\lfloor a^{<j}\right.$ and $\left.a^{>j}\right\rfloor$, so that there is no element left for alternatives in $A_{j}$, and representability is violated. This proves $\left\langle A_{j}\right\rangle \neq \emptyset$. Since the profile is weakly coherent by (i), using Lemma 3 (iii), we deduce that $\left\lfloor\stackrel{\circ}{A}_{j}\right\rceil \neq \emptyset$.

(iv) We know by (iii) that the interiors are not empty. A first fact to notice is that if we assume representability, we must have $\sharp\left[\left\lfloor A_{j^{\prime}}, A_{j}\right\rfloor\right] \geq j-j^{\prime}+1$, since this is the number of available elements on $E$ for assigning scores by a weak mean $G$ to classes $A_{j^{\prime}}$ to $A_{j}$ (included).

Let us assume that $\sharp\left[\left\lfloor A_{j^{\prime}}, A_{j}\right\rfloor\right]=j-j^{\prime}+1$, so that there is exactly one element on $E$ per class $A_{j^{\prime}}$ to $A_{j}$. Thus, if we denote by $a^{j}, a^{j^{\prime}}$ the alternatives in $A_{j}, A_{j^{\prime}}$ such that $\left.\left.a^{j}\right\rfloor=A_{j}\right\rfloor$ and $\left\lfloor a^{j^{\prime}}=\left\lfloor A_{j^{\prime}}\right.\right.$, necessarily for any $a \in A_{j}$ (resp. $a^{\prime} \in A_{j^{\prime}}$ ), $\left.G(a)=A_{j}\right\rfloor$ (resp. $G\left(a^{\prime}\right)=\left\lfloor A_{j^{\prime}}\right)$. Let us consider $j^{\prime \prime}<j$. Then for any $a \in A_{j^{\prime \prime}},\left\lfloor a<\left\lfloor a^{j^{\prime}}\right.\right.$, otherwise since $G$ is a weak mean operator, $G(a) \geq G\left(a^{j^{\prime}}\right)$, which violates the representation condition. This means that $\left\lfloor A_{<j^{\prime}}<\left\lfloor A_{j^{\prime}}\right.\right.$. Doing the same reasoning with $j^{\prime \prime}>j$, we obtain $\left.\left.A_{>j}\right\rceil>A_{j}\right\rfloor$. Hence $\sharp\left[\left\langle A_{j^{\prime}}, A_{j}\right\rangle\right] \geq j-j^{\prime}+1$, and by the assumption on $\sharp\left[\left\lfloor A_{j^{\prime}}, A_{j}\right\rfloor\right]$, we have $\sharp\left[\left\lfloor{\stackrel{\circ}{j^{\prime}}}^{\prime}, \stackrel{\circ}{j}_{j}\right\rceil\right]=j-j^{\prime}+1$.

Lastly, consider the case $\sharp\left[\left\lfloor A_{j^{\prime}}, A_{j}\right\rfloor\right]>j-j^{\prime}+1$. We denote by $\left\langle\left\langle A_{j^{\prime}}, A_{j}\right\rangle\right\rangle$ the interval of the scores assigned to classes $A_{j^{\prime}}, \ldots, A_{j}$, which is contained in $\left[\left\lfloor A_{j^{\prime}}, A_{j}\right\rfloor\right]$ ( $G$ is a weak mean operator). Remark that $\sharp\left\langle\left\langle A_{j^{\prime}}, A_{j}\right\rangle\right\rangle \geq j-j^{\prime}+1$. Doing the same reasoning as above, we obtain that $\left\lfloor A_{<j^{\prime}}<\left\langle\left\langle A_{j^{\prime}}\right.\right.\right.$, and $\left.\left.\left.A_{>j}\right\rceil>A_{j}\right\rangle\right\rangle$, hence the result.

Let us now suppose that $G$ is a mean operator. We have to show in addition that the profile is coherent. If $(A, \succsim, E)$ is not coherent, there exist $a^{\prime} \in A_{j^{\prime}}$ and $a \in A_{j}, j^{\prime}<j$, 
such that $a^{\prime} \geq a$. Since $G$ is a mean operator, this entails that $G\left(a^{\prime}\right) \geq G(a)$, and the representability condition does not hold.

$\Leftarrow)$ Let us suppose that the profile is weakly coherent and that (ii) to (iv) hold. We propose to construct $f$ and $E^{\prime}$ as follows (finest partition): for every non empty $K_{j}$, define $f\left(x_{j}\right)=e_{j}^{\prime}$ for all $x_{j} \in K_{j}$, ensuring $e_{j}^{\prime}<e_{j^{\prime}}^{\prime}$ whenever $j<j^{\prime}$, and otherwise take $f=I d$. Due to Lemma 3, we know that cores are disjoint and ordered the right way, hence the proposed construction is possible.

It remains to build $G$ being a weak mean operator. For each indifference class $A_{j}$ and each $a \in A_{j}$ we define $\left[\psi_{j}, \phi_{j}\right]$ to be the interval where $G$ can take its values for any $a \in A_{j}$. In order that $f \circ G$ represents $\succsim, f$ has to be constant over $\left[\psi_{j}, \phi_{j}\right]$.

We impose the following constraints on these intervals:

$(*)\left[\psi_{j}, \phi_{j}\right] \supseteq K_{j}, \forall j$ such that $K_{j} \neq \emptyset$.

$(* *)\left[\psi_{j}, \phi_{j}\right] \subseteq\left\lfloor\stackrel{\circ}{A_{j}}\right\rceil, \forall j$.

Condition $(*)$ is compatible with our above choice of $f$. Condition $(* *)$ is sound since $\left\lfloor\stackrel{\circ}{\left.A_{j}\right\rceil \neq \emptyset \text { by assumption }}{ }^{1}\right.$, and $\left\lfloor\stackrel{\circ}{A}_{j}\right\rceil \supseteq K_{j}$ (Lemma 4 (iv)). If $(* *)$ is not satisfied, it may exist an alternative $a$ from a class other than $A_{j}$ whose span is included in $\left[\psi_{j}, \phi_{j}\right]$, and since $G$ has to be internal, $G(a) \in\left[\psi_{j}, \phi_{j}\right]$, which entails $f \circ G(a)=f \circ G\left(a^{\prime}\right)$, for all $a^{\prime} \in A_{j}$.

The construction goes in several steps.

step 1: initialization of the intervals. For every $j \in\left\{1, \ldots, k^{\prime}\right\}$

1. If $K_{j} \neq \emptyset,\left[\psi_{j}^{0}, \phi_{j}^{0}\right]:=K_{j}$

2. Otherwise choose an element $e^{j}$ in $\left\lfloor\stackrel{\circ}{A}_{j}\right\rceil$ such that

2.1 If $K_{j+1} \neq \emptyset, e^{j}<\left\lfloor K_{j+1}\right.$, otherwise choose $e^{j+1}$ in $\left\lfloor A_{j+1}^{\circ}\right\rceil$ (if not already done) such that $e^{j}<e^{j+1}$.

2.2 If $\left.K_{j-1} \neq \emptyset, e^{j}>K_{j-1}\right\rfloor$, otherwise choose $e^{j-1}$ in $\left\lfloor A_{j-1}^{\circ}\right\rceil$ (if not already done) such that $e^{j}>e^{j-1}$.

\footnotetext{
${ }^{1}$ If condition (iii) is replaced by $\left\langle A_{j}\right\rangle \neq \emptyset$, then use weak coherence and Lemma 4 (iii) to infer the non emptiness of the interior.
} 
and let $\left[\psi_{j}^{0}, \phi_{j}^{0}\right]=\left\{e^{j}\right\}$. This family $\left\{\left[\psi_{j}^{0}, \phi_{j}^{0}\right]\right\}_{j=1, \ldots, k^{\prime}}$ of disjoint sets is called a pre-partition of $E$.

We have to show that such a construction is possible. Condition 1 has already been examined above (cores are disjoint and properly ordered). For condition 2 , if $K_{j+1} \neq \emptyset$, from Lemma 2 (iii), it is possible to find $e^{j} \in\left\lfloor\stackrel{\circ}{A_{j}}\right\rceil$ to the left of $K_{j+1}$, and similarly for $K_{j-1}$. If $K_{j+1}=\emptyset$, from (iii) and Lemma 2 (ii), we know that at worst the interiors $\left\lfloor A_{j}\right\rceil$ and $\left\lfloor A_{j+1}^{\circ}\right\rceil$ coincide (otherwise $\left\lfloor A_{j+1}^{\circ}\right\rceil$ has its bounds greater than those of $\left\lfloor A_{j}\right\rceil$, and $e^{j}$ and $e^{j+1}$ can be found). In this case, if $\sharp\left\lfloor\stackrel{\circ}{A}_{j}\right\rceil>1$ we are done. The case $\sharp\left\lfloor A_{j}\right\rceil=1$ is forbidden by condition (iv) of the theorem. Now, from condition (ii), there is always enough elements between the cores to assign elements to classes. From condition (iv), between the boundaries of any two interiors $\left\lfloor\stackrel{\circ}{A}_{j}\right\rceil,\left\lfloor\stackrel{\circ}{A_{j^{\prime}}}\right\rceil$, there will be always enough elements.

step 2: construction of the intervals. We build first the intervals $\left[\psi_{j}^{1}, \phi_{j}^{1}\right]$ for all $j$ as follows:

$$
\left.\left[\psi_{j}^{1}, \phi_{j}^{1}\right]:=\right] \phi_{j-1}^{0}, \psi_{j+1}^{0}\left[\cap\left\lfloor\stackrel{\circ}{A_{j}}\right]\right.
$$

These intervals are not empty, since by construction $\phi_{j-1}^{0}<e^{j}<\psi_{j+1}^{0}$, and $e^{j} \in\left\lfloor\stackrel{\circ}{j}_{j}\right\rceil$. Note that these intervals may intersect (for two adjacent $j, j^{\prime}$ only), but they are ordered in a strictly increasing way, i.e. $j^{\prime}<j$ implies $\psi_{j^{\prime}}^{1}<\psi_{j}^{1}$ and $\phi_{j^{\prime}}^{1}<\phi_{j}^{1}$ since all the $\left[\psi_{j}^{0}, \phi_{j}^{0}\right]$ are disjoint. We eliminate all common parts, by cutting every non empty intersection in two parts, one of them possibly empty.

Suppose that $\left[\psi_{j}^{1}, \phi_{j}^{1}\right] \cap\left[\psi_{j+1}^{1}, \phi_{j+1}^{1}\right]=: B_{j} \neq \emptyset$, and $\sharp B_{j}=: b_{j}>0$. Because of strict increasingness of the intervals, $B_{j}$ cannot be equal to one of these intervals. There are $b_{j}+1$ different ways to cut $B_{j}$ in two parts, including at most one empty part, attributing the left one to $\left[\psi_{j}^{1}, \phi_{j}^{1}\right]$, and the right one to $\left[\psi_{j+1}^{1}, \phi_{j+1}^{1}\right]$. Let us denote these new intervals by $\left[\psi_{j}, \phi_{j}\right]$, now disjoint, and non empty.

step 3: construction of $G$. For any $a_{j} \in A_{j}, \forall j=1, \ldots, k^{\prime}$, we have to define $G$ such that $G\left(a^{j}\right)$ be any element in $\left[\psi_{j}, \phi_{j}\right] \cap\left\lfloor a^{j}\right\rfloor$. Let us show first that this intersection is never empty. Indeed:

- $\left[\psi_{j}, \phi_{j}\right]$ is not empty. 
- $\left[\psi_{j}, \phi_{j}\right] \cap\left\lfloor a^{j}\right\rfloor$ is not empty. Let us show that $\left[\psi_{j}^{1}, \phi_{j}^{1}\right] \cap\left\lfloor a^{j}\right\rfloor \neq \emptyset$, which amounts to show that $] \phi_{j-1}^{0}, \psi_{j+1}^{0}\left[\cap\left\lfloor a^{j}\right\rfloor \neq \emptyset\right.$, and $\left\lfloor\stackrel{\circ}{A}_{j}\right\rceil \cap\left\lfloor a^{j}\right\rfloor \neq \emptyset$. The first fact is shown as follows. Let us suppose that the intersection is empty, and that $\left\lfloor a^{j}\right\rfloor$ is to the left of ]$\phi_{j-1}^{0}, \psi_{j+1}^{0}\left[\right.$ (the reasoning is much the same in the symmetrical case). $\phi_{j-1}^{0}$ is equal to either $e^{j-1}$ if $K_{j-1}=\emptyset$, or $\left.K_{j-1}\right\rfloor$. In the first case, we have $\left.\left.a^{j}\right\rfloor \leq e^{j-1} \leq \stackrel{\circ}{A-1}_{j}\right\rfloor$, which contradicts the definition $\left.\stackrel{\circ}{j-1}_{j}\right\rceil$ (more precisely, of $\left.A_{>j-1}\right\rceil$ ). In the second case, denoting $a^{j-1}$ an alternative of $A_{j-1}$ such that $\left\lfloor a^{j-1} \geq K_{j-1}\right\rfloor$, we would have $a_{i}^{j} \leq a_{i}^{j-1}$, for all $i$, which violates coherence since $a^{j} \succ a^{j-1}$. The second fact is due to (iii) and Lemma 4 (ii). Now observe that the (possible) intersection part between the two intervals $\left[\psi_{j}^{1}, \phi_{j}^{1}\right]$ and $\left[\psi_{j+1}^{1}, \phi_{j+1}^{1}\right]$ is contained in $\left\lfloor\stackrel{\circ}{A_{j}}\right\rceil \cap\left\lfloor A_{j+1}^{\circ}\right\rceil$. Since no $a \in A$ can be such that $\lfloor a\rfloor \subseteq\left\lfloor\stackrel{\circ}{A}_{j}\right\rceil \cap\left\lfloor A_{j+1}^{\circ}\right\rceil$, no splitting of the intersection can isolate $\left\lfloor a^{j}\right\rfloor$, so that $\left\lfloor a^{j}\right\rfloor \cap\left[\psi_{j}, \phi_{j}\right] \neq \emptyset$

It remains to choose a value in the intersection. If $A_{j}$ contains a non empty core $K_{j}$, by construction $K_{j} \subseteq\left[\psi_{j}, \phi_{j}\right]$. Moreover, any $a \in A_{j}$ intersects $K_{j}$ since $K_{j}$ is the interval between the two "extreme" (most disjoint) individuals in $A_{j}$. Hence it is possible to define:

$$
G(a) \in K_{j} \cap\lfloor a\rfloor
$$

Suppose on the contrary that $A_{j}$ has an empty core. Then $G$ has to be constant over $A_{j}$. We are done if we show that there exists $x_{j} \in\left[\psi_{j}, \phi_{j}\right]$ belonging to the span of each $a \in A_{j}$, hence to $\cap_{a \in A_{j}}\lfloor a\rfloor$, since in this case it suffices to put $G(a)=x_{j}$ for all $a \in A_{j}$.

We have proved above that $\left[\psi_{j}, \phi_{j}\right] \cap\lfloor a\rfloor \neq \emptyset$, for all $a \in A_{j}$. Suppose there is no common point of intersection in the interval $\left[\psi_{j}, \phi_{j}\right]$. This would mean that it exist two alternatives $a, a^{\prime} \in A_{j}$ with disjoint spans. But this contradicts the fact that $K_{j}=\emptyset$.

$G$ is indeed a weak mean operator, since $G(a)$ is in the span of a (clear from the definition of $G$ ). Clearly $f \circ G$ represents $\succsim$.

Let us assume now that the profile is coherent. In step 3, we impose in addition the following:

$$
\forall a, b \in A_{j} \text { such that } a_{i} \geq b_{i}, \forall i, \quad G(a) \geq G(b)
$$

Indeed, strict inequality for $G$ happens only when $A_{j}$ has a non empty core, where $G$ can be defined so as to satisfy (2). 
Let us show that $G$ is non decreasing over $A$. Let us take $a, a^{\prime} \in A$ such that $a_{i} \geq a_{i}^{\prime}$, $\forall i . a^{\prime}$ is necessarily either in the same class than $a$ (say $A_{j}$ ), or in a lower class (say $A_{j^{\prime}}$,

$j^{\prime}<j$ ). In the first case, equation (2) ensures that $G(a) \geq G\left(a^{\prime}\right)$. In the second case, $G\left(a^{\prime}\right) \in\left[\psi_{j^{\prime}}, \phi_{j^{\prime}}\right]$, which is an interval to the left of $G(a)$, and the result holds.

The case of strong and strict coherence proceeds similarly.

\section{References}

[1] D. Bouyssou and M. Pirlot. Preferences for multi-attributed alternatives: traces, dominance, and numerical representations. J. of Mathematical Psychology, 48:167-185, 2004.

[2] A. Cauchy. Cours d'Analyse de l'École Royale Polytechnique. Première Partie : Analyse algébrique. Paris, 1821.

[3] D. Dubois, H. Prade, and R. Sabbadin. Decision-theoretic foundations of qualitative possibility theory. Eur. J. of Operational Research, 128:459-478, 2001.

[4] H. Fargier and R. Sabbadin. Qualitative decision under uncertainty: back to expected utility. Artificial Intelligence, 164:245-280, 2005.

[5] P. Fishburn. Interval orders, interval graphs - A study of partially ordered sets. J. Wiley \& Sons, 1985.

[6] J. Fodor. Smooth associative operations on finite ordinal scales. IEEE Tr. on Fuzzy Systems, 8(6):791-795, 2000.

[7] M. Grabisch. The application of fuzzy integrals in multicriteria decision making. European J. of Operational Research, 89:445-456, 1996.

[8] M. Grabisch. Alternative representations of discrete fuzzy measures for decision making. Int. J. of Uncertainty, Fuzziness, and Knowledge Based Systems, 5:587-607, 1997.

[9] M. Grabisch. Identification of mean aggregation operators on finite scales. working paper, 2004.

[10] M. Grabisch, Ch. Labreuche, and J.C. Vansnick. On the extension of pseudo-Boolean functions for the aggregation of interacting bipolar criteria. Eur. J. of Operational Research, 148:28-47, 2003. 
[11] D.H. Krantz, R.D. Luce, P. Suppes, and A. Tversky. Foundations of measurement, volume 1: Additive and Polynomial Representations. Academic Press, 1971.

[12] J.-L. Marichal and R. Mesiar. Aggregation on finite ordinal scales by scale independent functions. Order, 21:155-180, 2004.

[13] J.-L. Marichal, R. Mesiar, and T. Rückschlossová. A complete description of comparison meaningful functions. Aequationes Mathematicae, 69:309-320, 2005.

[14] J.L. Marichal. An axiomatic approach of the discrete Choquet integral as a tool to aggregate interacting criteria. IEEE Tr. on Fuzzy Systems, 8(6):800-807, 2000.

[15] J.L. Marichal. On Sugeno integral as an aggregation function. Fuzzy Sets and Systems, 114:347-365, 2000.

[16] J.L. Marichal. An axiomatic approach of the discrete Sugeno integral as a tool to aggregate interacting criteria in a qualitative framework. IEEE Tr. on Fuzzy Systems, 9(1):164-172, 2001.

[17] M. Mas, G. Mayor, and J. Torrens. T-operators and uninorms on a finite totally ordered set. Int. J. Intelligent Systems, 14(9):909-922, 1999.

[18] M. Mas, M. Monserrat, and J. Torrens. On bisymmetric operators on a finite chain. IEEE Tr. on Fuzzy Systems, 11(5):647-651, 2003.

[19] S. Ovchinnikov. Means on ordered sets. Mathematical Social Sciences, 32:39-56, 1996.

[20] A. Rico, M. Grabisch, Ch. Labreuche, and A. Chateauneuf. Preference modelling on totally ordered sets by the Sugeno integral. Discrete Applied Maths., 147:113-124, 2005.

[21] M. Sugeno. Theory of fuzzy integrals and its applications. PhD thesis, Tokyo Institute of Technology, 1974. 